\title{
René Maran lecteur de Charles Darwin et Rémy de Gourmont
}

\author{
Roseline Garcia Ballester
}

\section{OpenEdition \\ Journals}

Édition électronique

URL : https://journals.openedition.org/coma/7600

DOI : $10.4000 /$ coma.7600

ISSN : 2275-1742

\section{Éditeur}

Institut des textes \& manuscrits modernes (ITEM)

\section{Référence électronique}

Roseline Garcia Ballester, «René Maran lecteur de Charles Darwin et Rémy de Gourmont », Continents manuscrits [En ligne], 17 | 2021, mis en ligne le 15 octobre 2021, consulté le 08 janvier 2022. URL : http://journals.openedition.org/coma/7600 ; DOI : https://doi.org/10.4000/coma.7600

Ce document a été généré automatiquement le 8 janvier 2022.

\section{(C) $\odot \Theta \Theta$}

Continents manuscrits - Génétique des textes littéraires - Afrique, Caraîbe, dispora est mis à disposition selon les termes de la licence Creative Commons Attribution - Pas d'Utilisation

Commerciale - Pas de Modification 4.0 International. 


\title{
René Maran lecteur de Charles Darwin et Rémy de Gourmont
}

\author{
Roseline Garcia Ballester
}

De cet état de dépression, n'allez pas déduire que je me porte mal. Au contraire, ma santé est excellente ${ }^{1}$.

$1 \quad$ Il s'agit, dans ce développement, d'interroger un aspect récurrent de l'œuvre de René Maran, non exploré jusque-là : l'influence des théories de Charles Darwin sur la construction des personnages et de l'intrigue romanesques.

2 Il faut remarquer tout d'abord que René Maran ne cite pas le nom de Darwin. Mais il fait régulièrement référence à un petit essai publié en 1903 pour la première fois par Rémy de Gourmont : Physique de l'amour, sous-titré Essai sur l'instinct sexuel. Or, l'objectif de Gourmont est de commenter les théories du savant anglais, dans cent cinquante-six pages denses et documentées ${ }^{2}$. Pour cela, il reprend à son compte la notion d'instinct, fil rouge qui organise les comptes rendus des observations et analyses de Darwin. René Maran a recours, après Rémy de Gourmont, à la notion d'instinct qui prend dans son texte des colorations différentes, en fonction de l'intention qui lui donne forme.

Cette intention doit être prise en considération en rapport avec la violence coloniale à laquelle est confronté l'auteur dans le cadre de ses fonctions d'administrateur en Afrique équatoriale française (AEF).

Retraçons tout d'abord les grandes lignes de la théorie de Charles Darwin que discute Rémy de Gourmont. Nous examinerons ensuite quelques-unes des pistes proposées par l'œuvre de René Maran pour cerner son apport à la notion d'instinct. 


\section{Théories de l'instinct dans la bibliothèque de René Maran}

\section{Les théories darwiniennes}

5 Le premier essai de Charles Darwin, L'Origine des espèces, publié pour la première fois en 1859, met en forme une partie des très nombreuses notes prises à bord du Beagle, pendant un voyage de quatre années. Le naturaliste anglais fait le tour du monde. Il observe, note, dessine, croque. Toutes les espèces vivantes retiennent son intérêt, de la plus « archaïque » à la plus «sophistiquée », l'homme compris. La notion d'instinct, centrale pour expliquer les mécanismes de transformations au cours de la succession des générations, n'est pas clairement définie par Darwin, mais serait toutefois à l'origine de ces transformations. Par-là, il réfute la notion de fixisme défendue par la doxa religieuse, ce qui, dans un premier temps, fait scandale comme on le sait.

Ce que nous prenons pour des habitudes, explique Darwin, ne serait en fait que la traduction des instincts positifs transmis de générations en générations. Les espèces vivantes, en effet, seraient prédisposées pour conserver ce qui leur permet d'accroître leurs capacités à perdurer dans leur être. En ce sens, les lois morales, les sentiments d'empathie ou de solidarité entre les membres d'une même espèce, s'exerceraient dans un but strictement utilitaire. Dans la même veine, l'idée de liberté serait illusoire, soutient Darwin, née de spéculations sans lien avec le réel : ce que nous appelons liberté ne peut s'exercer qu'à l'intérieur d'un éventail de possibilités limitées, déterminées par la morphologie et la physiologie de l'individu concerné. La liberté serait donc un choix contraint par la seule nécessité de survivre.

7 Pour Darwin, toutes les espèces, aussi rudimentaires soient-elles, se transforment et changent au fil des générations. Elles se transforment puisqu'elles accumulent, dans le temps, des dispositions positives. Pour pouvoir ainsi décider de ce qui est positif, c'està-dire qui permet de se protéger et se prémunir d'un danger, il faut bien qu'elles possèdent une intelligence. En ce sens, instinct et intelligence ne s'opposent pas, mais, au contraire, se complètent : également indispensables l'une et l'autre et l'une à l'autre à la perpétuation de l'espèce. Dans cette perspective, animalité et humanité s'envisagent sur un même plan.

8 Tout individu dispose d'une conscience qui lui permet de décider de ce qui lui est profitable ou non, ou plus ou moins profitable. Tout individu, selon la théorie évolutionniste, s'adapte de manière toujours plus performante à son environnement. L'idée de sélection naturelle permet à Darwin, lecteur de Malthus ${ }^{3}$, d'expliquer la coexistence d'espèces diverses sur un même espace avec des ressources limitées : seuls survivent les meilleurs, les plus adaptés. En même temps, toute vie qui se déploie dans l'espace-temps ignore à quel schème premier, primordial, elle répond. Les générations se succèdent dans l'ignorance de ce qu'elles mettent en œuvre pour rendre leur succession possible. Le mécanisme fondamental de la vie est inconscient; toute vie ne peut être que dans la non-connaissance de ce qui la fait être, dans l'ignorance de sa genèse. 


\section{À l'origine de la notion d'instinct dans les textes de Maran : La Physique de l'amour de R. de Gourmont}

Les personnages des nouvelles et romans de René Maran « ont » dans leur bibliothèque le petit essai Physique de l'amour de Rémy de Gourmont, un auteur que René Maran convoque régulièrement. Ainsi par exemple dans une lettre envoyée à Manoel Gahisto, le 11 janvier 1916 de Bordeaux :

L'animalité me plait autant qu'elle pouvait agréer à Rémy de Gourmont. Comme vous, au reste, j'essayais d'y ramener tous les actes humains ${ }^{4}$.

Manoel Gahisto et René Maran sont donc d'accord pour considérer Rémy de Gourmont comme une référence. Or si Rémy de Gourmont partage la vision mécaniste et progressive de Charles Darwin, il conteste la théorie du naturaliste anglais sur au moins deux points essentiels : la hiérarchisation des espèces d'une part; et le statut de la sexualité d'autre part. René Maran partage la critique de Rémy de Gourmont; les commentaires à son ami Manoel Gahisto, dans la correspondance, le confirment.

\section{L'évolution en question}

11 Concernant la hiérarchie des espèces, Rémy de Gourmont remet vivement en question les degrés évolutionnistes dont rendent compte les schémas darwiniens.

Darwin est venu, et il a inauguré une méthode utile, mais ses vues sont trop systématiques, son but trop explicatif, son échelle des êtres, avec l'homme en haut, somme de l'effort universel, d'une simplicité trop théologique. L'homme n'est pas au sommet de la nature; il est dans la nature, une des unités de la vie, et rien de plus ${ }^{5}$.

12 L'auteur de Physique de l'amour considère un jaillissement de multiples formes de vies, indépendantes les unes des autres dans leur évolution. Chaque espèce du règne animal et végétal s'organise et progresse en fonction d'elle-même et de la pertinence qui la caractérise. Contre un modèle hiérarchique de la pensée, Rémy de Gourmont défend un modèle horizontal de singularités qui cohabitent et défient l'Un, pour reprendre la terminaison glissantienne ${ }^{6}$. Il n'est nullement avisé de classer selon un ordre de valeurs posées par les hommes et qui n'a aucun sens du point de vue de la manifestation des phénomènes. Non seulement l'esprit de hiérarchie est indifférent au règne du vivant mais il nous éloigne de son intelligence. Puisqu'il faut penser le vivant sur un plan horizontal, il faut bien considérer l'homme et l'animal sur le même plan. Rémy de Gourmont radicalise la mise en question de la supériorité communément admise de l'homme sur l'animal, que consacre Darwin dans son modèle évolutionniste. L'instinct, moteur commun aux deux espèces, détermine les possibilités d'existence sur la terre. Il se distingue, dans ses différentes manifestations, selon les besoins physiologiques des uns ou des autres. Animaux ou humains sont assignés aux mêmes impératifs.

La distinction, à ce point de vue, entre l'homme et les animaux, est de quantité et non de qualité. Il ne faut pas se laisser duper par la distinction scolastique entre l'instinct et l'intelligence : l'homme est tout aussi chargé d'instinct que l'insecte le plus instinctif?

En ce sens, ce que nous appelons l'instinct animal reste enfoui dans l'homme, se frayant à la lumière d'un évènement ou d'un autre, un chemin jusqu'à la conscience dont il brise la résistance. 
Quant à René Maran, lecteur fidèle des œuvres de Rémy de Gourmont, il multiplie ses recours au mot instinct, dans les écrits fictionnels, autobiographiques et les articles publiés dans différentes revues dont nous disposons. Il s'impose donc de distinguer les différents sens et fonctions qu'il lui accorde, différents les uns des autres, selon leur position dans l'avant-texte des correspondances et leur transposition dans les écrits publiés. Lors de sa première mutation sur un poste en brousse, René Maran, épris de solitude, en est ravi. Voici ce que l'on peut lire dans une lettre envoyée à Manoel Gahisto :

C'est la vie lumineuse, animale, instinctive. Je ne sais pas pourquoi, dans la psychologie actuelle, l'instinct est presque le synonyme d'ombre. C'est tout le contraire. Saisissez-vous bien toute l'analogie qu'il y a entre l'instinct et la lumière.

Ils sont tous deux originels... ${ }^{8}$

Les adjectifs lumineuse et instinctive sont soulignés par l'auteur. L'instinct est du côté de la lumière. Et non la raison, comme l'ont soutenu les philosophes des Lumières... D'une part, la tradition humaniste dont Maran est familier, notamment par sa fréquentation des philosophes de l'Antiquité, doit être discutée en ce sens qu'elle ne nous permettrait pas de penser la diversité des cultures. D'autre part, il s'en prend au dualisme entretenu par certains intellectuels de son époque : l'instinct est associé au sauvage, la raison à la civilisation ${ }^{9}$. Il opère donc un renversement épistémologique. Néanmoins, la notion d'instinct, chez Maran, n'apparaît pas toujours lié à cette vie lumineuse. Ce que René Maran appelle instinct se charge des ressentis intimes les plus divers, selon son état d'esprit du moment, et suffit, du moins dans l'ordre du langage, à rendre compte de tout ressenti. Sa conception de l'instinct le conduit à intimement lier les genres animal et humain. L'écrivain en expose les tenants et aboutissants à travers ses correspondances et dans ses fictions.

\section{L'égalité des espèces humaines et animales}

16 On sait que René Maran faisait preuve d'un véritable intérêt pour les mœurs des animaux. La lettre du $1^{\mathrm{er}}$ août 1912, envoyée de Grimari à Manoel Gahisto, fait mention $\mathrm{du}$ nom de Jean Marie-Fabre, entomologiste dont le travail anticipe l'éthologie du $\mathrm{xx}^{\mathrm{e}}$ siècle. Les animaux tiennent une place importante dans la vie des personnages de ses nouvelles françaises et africaines (pour reprendre la dénomination de Roger Little ${ }^{10}$ ). Ils sont le double de l'être humain, sa conscience, son prolongement, son unique consolation. Autrement dit, l'homme projette sur l'animal, au sens que donne Freud à ce terme. L'écrivain en revient toujours à l'animal, personnage central à partir duquel se fait l'approche de la psychologie du personnage masculin ou féminin.

Lors de ses séjours en Oubangui-Chari, René Maran considère comme des compagnons à part entière les animaux qui l'entourent. Pour eux, il défend une véritable théorie éthique dans laquelle des mouvements antispécistes contemporains pourraient se reconnaître.

18 Dans une lettre datée du 11 octobre 1912, René Maran explique avoir sauvé un homme piqué par un serpent noir à l'aide du sérum Palmette, antivenimeux dont on dispose alors en Oubangui-Chari, et aussi d'une infusion de citronnelle, jugée excellent fébrifuge ${ }^{11}$.

Je bénis le Dr Palmette, dont la science m'a indirectement guéri de ma tristesse de ce matin en me procurant une des joies les plus pures et les plus grandes de ma vie, la joie d'arracher un homme à l'envahissant crépuscule de la mort. Bonheur ${ }^{12}$. 
Un peu plus loin, toujours dans la même lettre, il écrit :

Cependant, j'ai eu une joie à peu près égale l'autre jour. Il y avait un pauvre cabri, pas encore adulte, qui, météorisé par l'herbe mouillée du matin, avait une de ces diarrhées qui vous enlèvent un ruminant en deux jours. Je lui ai fait faire une place dans mon poulailler... Maintenant, il bêle et vient me lécher la main quand il me voit. Je n'ai pas l'âme sensible de Fénelon ni des bergères de Marie-Antoinette. Mais cela me fait plaisir et m'émeut profondément... ${ }^{13}$

Outre l'intérêt qu'il porte à la médecine locale, ces passages épistolaires nous renseignent sur les convictions de René Maran : une vie animale vaut une vie humaine. Les émotions suscitées chez lui par les évènements qu'il relate sont équivalentes, quels que soient les êtres vivants concernés, animal ou humain. Dans un autre passage, pris cette fois dans une lettre du 24 mars 1917, on peut lire :

Je m'analyse. Sociable? Je le suis au sens le plus élevé du terme. Et, cependant, la société m'écœure, la société humaine. Je ne dis pas que les animaux sont nos frères inférieurs. Mais je témoigne à certaines bêtes, aux chiens par exemple, une amitié qui va jusqu'aux larmes ${ }^{14}$.

Quant aux héros de L'homme qui attend, modeste employé parisien, il emmène avec lui dans la mort son petit chat, compagnon avec lequel il vit de façon fusionnelle depuis le départ de sa femme :

Je m'en voudrais de laisser mon chat à des indifférents. Il en mourrait de chagrin. Mieux vaut le piquer n'est pas? Ainsi la pauvre bête ne souffrira plus. Je pourrai m'en aller en paix.... Sa voix s'étrangla. Il était à bout de forces, à bout de chagrin ${ }^{15}$.

Il convient d'évoquer aussi Le Livre de la brousse, œuvre d'une extraordinaire complexité. À la façon des fables de La Fontaine, l'un des auteurs que René Maran admirait, toutes les espèces "parlent » et se comprennent, dans la violence d'une nature qui s'impose impitoyable aux uns et aux autres. Il faudrait tout un développement pour rendre compte de la relation complexe du héros, Kossi, avec la panthère Moura, personnage qui revient régulièrement tant dans les nouvelles que dans ses confidences à Gahisto. Au terme d'un combat terrible, Moura est tuée par Kossi, qui entend défendre sa vie et celle de ses chiens. L'agonie de Moura précède de peu la mort de la compagne de Kossi et de leur bébé. Elle précède aussi celle de Kossi qui l'entraine avec lui dans la mort. Les scènes parisienne et africaine se répondent ainsi, l'homme et l'animal, comme son double, devant périr dans le même combat final. Mais dans ce dernier combat, l'héroïsme tragique du héros est du côté africain.

Dans la relation de l'homme à l'animal, chacun évoluant dans un même espace, hommes et animaux sont liés dans la finitude de la vie. Dans la nouvelle L'homme qui attend, la mort délivre le personnage de la souffrance de vivre; il emmène avec lui son chat fidèle, dans ce terrible geste que les psychiatres qualifient aujourd'hui de suicide altruiste :

On les retrouva, Miaou et lui, à peu près dans la même position, le lendemain matin, vers midi, quand on eut forcé sur la demande de sa femme, la porte de leur appartement.

Il s'était enfin changé en lui-même, n'attendait plus personne et semblait sourire ${ }^{16}$.

23 La pulsion de destruction renvoie toute vie à l'état de non-vie qui l'a précédée, explique Freud. Dans ce retour ultime, L'homme qui attend révèle la force du fantasme de fusion avec l'Autre qui l'habitait. Peut-être seul l'animal pouvait-il le suivre jusque-là... 


\section{Les derniers mots de l'instinct sexuel} qui concerne l'instinct sexuel, des distinctions fondamentales de comportements entre les genres et entre les individus d'un même genre. Le domaine des compétitions sexuelles devient le seul dans lequel se manifeste des inégalités entre mâles et femelles (celui de la survie par exemple, ignorant la distinction de genre). La notion d'avantages secondaires permet à Darwin de rendre compte de l'existence de traits qui ne relèvent plus absolument de l'ordre de l'obligatoire. Par exemple, la masse de ses bois, pour le cerf, est un avantage d'ordre purement esthétique. Alors que la lourdeur peut le désavantager au moment de combattre face à un adversaire, l'aspect esthétique prime dans la compétition puisqu'il permet de retenir l'attention des femelles et donc leur consentement pour l'accouplement. Car si les avantages secondaires ne sont pas directement liés aux mécanismes de la reproduction sexuelle, ils ne leur sont pas non plus complètement étrangers. Ils augmentent les chances de s'assurer une descendance. Cependant, il faut bien remarquer, comme l'écrit Patrick Tort, spécialiste de l'œuvre de Darwin, que la volonté de séduire implique précisément volonté:

On remarquera ici dans le texte de Darwin un infléchissement remarquable qui parait assigner à la conquête du partenaire sexuel et reproductif une participation plus sensible de la volonté consciente des individus ${ }^{18}$.

On passe, précise-t-il encore, et selon une belle expression « du commerce des armes à celui du charme ».

(...) ces deux expressions s'équivalent parce qu'elles désignent d'une manière parfaitement interchangeable les mêmes instruments de la conquête amoureuse ${ }^{19}$.

C'est précisément sur ce point de la théorie que Rémy de Gourmont et René Maran se désolidarisent, chacun à sa façon, de Charles Darwin.

\section{Le fait sexuel : désaccords avec Charles Darwin}

Rémy de Gourmont souligne l'inachèvement du travail mené par Darwin pour proposer une explication scrupuleusement matérialiste de toute forme du vivant. Pour de Gourmont, il s'agit d'une régression, d'un renoncement, qu'il évoque en ces termes :

D'ailleurs Darwin, soumis à la pudibonderie religieuse de sa race a presque entièrement négligé les faits sexuels stricts, et cela rend incompréhensible sa théorie de la sélection sexuelle comme principe de changement ${ }^{20}$.

Rémy de Gourmont veut rester étranger à la subtilité du lien tissé par Darwin entre l'apparition concomitante, chez certaines espèces sophistiquées, des facultés de 
vouloir, de désirer, et du sentiment esthétique. Pour lui, le désir sexuel s'affirme en tant que tel, sans autre finalité que de tendre à sa satisfaction.

Qu'il soit chaste ou voluptueux, avare ou prodigue de sa chair, l'homme n'en est pas moins, en tout état, soumis à la tyrannie de la chair ${ }^{21}$.

Le libertaire aristocrate que fut Rémy de Gourmont compose avec, ou même célèbre, la tyrannie de la chair. Il trouve, malgré son visage ravagé par le lupus qui le tient cloitré dans son appartement parisien, des muses au service de son talent. L'auteur de Physique de l'amour fait de son handicap la marque singulière d'un dandy hors norme : il confie à l'œuvre le soin de « réparer » son corps difforme. Il se confie à l'écriture qui lui permet de sublimer, au sens que Freud donne à ce verbe.

31 L'auteur de Batouala considère lui aussi la tyrannie de la chair qui tient sous sa coupe l'humanité; il l'évoque sous les signes de la mélancolie qui caractérise son fonctionnement psychique et qu'il veut ainsi justifier :

L'amour qui dirige le monde n'est que la forme sexuelle de l'intérêt, de l'intérêt supérieur qui veut que la vie ait, pour se transmettre d'âge en âge jusqu'à la fin des temps, ses porteurs de flambeaux ${ }^{22}$.

Quand il s'agit de l'instinct sexuel, René Maran ne manque pas une occasion de se libérer du ressentiment qu'il nourrit contre le genre féminin. Dans la lettre de 1916, déjà évoquée plus haut, il est question de la période de la guerre qui permet de découvrir le « vrai » visage des femmes :

Quoi, la femme monogame! Hypocrisie et dérision. On se jette au cou du premier homme qui passe, trop jeune ou trop vieux, si malade qu'il puisse être. L'instinct de conservation de la race, dont parlait Schopenhauer, ne préside que peu à ces accouplements précipités où il n'y entre nulle sympathie, nulle affection...23

C'est à Schopenhauer, autre auteur auquel il fait référence dans sa correspondance, que René Maran demande ici, bien facilement, appui. Pendant ce temps, explique encore l'écrivain au destinataire de la lettre, les hommes sont retenus par leurs devoirs, qu'ils accomplissent au risque de leur vie. Les hommes sont donc capables d'un refoulement de leurs instincts primaires pour répondre à des intérêts supérieurs, mécanisme de sublimation inaccessible aux femmes - et aux «primitifs » en tant que sujets mineurs. Dans une autre lettre, envoyée celle-ci à son ami Charles Barrailley, un an après environ :

Cette guerre fera comprendre aux hommes, s'ils l'ignoraient encore, que les

femmes sont esclaves, comme nous, de leurs appétits sexuels...24

L'instinct explique donc, selon lui, les comportements humains. Il est la clé pour comprendre les orientations, orientations qui tendent toujours vers la trahison, la dégradation...

\section{En guise d'ouverture}

Les réflexions circonstanciées rapportées ci-dessus ne suffisent pas à épuiser le rapport problématique entretenu par René Maran à l'élément féminin, dont les causes selon lui sont à chercher dans la différence de couleur de peau dans le couple. Les échecs sentimentaux qu'il subit plongent René Maran dans la mélancolie, état psychique permanent qui trouve là sa justification. La rupture avec Bernerette, sœur de l'épouse de Manoel Gahisto, alors qu'il avait conçu des projets de mariage, le plonge pendant 
plusieurs années dans un désespoir absolu. Il est envahi d'un vécu morbide et l'écrit, d'Afrique, à Monsieur et Madame Gahisto qui s'alarment.

Peut-être ne me reverrez-vous plus jamais. Il faut souhaiter cela. La mort serait pour moi une délivrance. Si vous saviez comme je suis lassé de tout et de moimême ${ }^{25}$.

Il faudra accorder à la complexité du ressenti du personnage de Jean Veneuse, investi par René Maran comme son propre reflet, le long développement qu'elle mérite, à l'appui des connaissances cliniques dont nous disposons aujourd'hui. Ce travail sera en effet très producteur pour une connaissance approfondie de l'œuvre. Frantz Fanon, psychiatre de formation, dans le chapitre de Peau noire masques blancs intitulé "L'homme de couleur et la blanche ${ }^{26}$ » avance les arguments convaincants que l'on sait. Comme le fait remarquer Matthieu Renaud, le jugement sans appel que le scientifique porte sur l'homme-écrivain, confondu alors avec son personnage principal, porte quelque peu ombrage à leur pertinence ${ }^{27}$. Il n'en demeure pas moins que le recours à la clinique de la névrose d'abandon ${ }^{28}$, que connaissait Fanon et qu'il considère dans ce chapitre de Peau noire, masques blancs, peut éclairer les contradictions de René Maran. La façon dont le personnage-écrivain d'Un homme pareil aux autres se heurte à la problématique coloniale de la « race » atteste de l'intériorisation d'un tabou racial lié à la situation coloniale.

Car je ne suis qu'un nègre, moi. Et un nègre n'a pas le droit de s'évader de sa race ${ }^{29}$.

37 En même temps, l'insistance avec laquelle la plainte revient et revient, le fait même qu'il y ait formalisation de la plainte, invite à la considérer avec circonspection. Posture pour camoufler le vertige d'un désir troublant?

Il faudrait examiner à quel point et comment René Maran se donne et nous donne les clés pour examiner la complexité du vécu de Jean Veneuse. Le recours au mot de dépersonnalisation, qu'emploie le personnage, témoigne de la prise de conscience aigüe de son malaise. Le concept est emprunté au philosophe, spécialiste de la conscience, Ludovic Dugas, auteur de La Mémoire et l'Oubli, dont René Maran possédait un exemplaire dans sa bibliothèque :

Je suis là, dans cette foule, moi, Jean Veneuse. J'assiste à mon propre départ. Je me suis dédoublé, dépersonnalisé ${ }^{30}$.

39 La question qui se pose alors est celle de la qualité de l'investissement du réel, que l'auteur traduit par le terme de dépersonnalisation. En quoi le vécu de dépersonnalisation diffère-t-il du concept d'inquiétant étrange que développe Freud ? Pourquoi vient-il ici renforcer le mot dédoublé qui peut renvoyer à l'idée de double conscience noire, théorisée par William Dubois (que lisait aussi René Maran) ? Quels liens ces termes entretiennentils avec le désir de Jean Veneuse, quittant Bordeaux ${ }^{31}$ et s'éloignant ainsi de la femme convoitée, à la fois avec regret et soulagement?

40 Achille Mbembe ouvre d'inestimables pistes de réflexion dans son ouvrage Politiques de l'inimitié. Il écrit :

L'entreprise coloniale tirait une grande partie de sa substance et de son excès d'énergie de son lien avec toutes sortes de flux pulsionnels, de désirs plus ou moins avoués dont la plupart se situaient en deçà du moi conscient des acteurs concernés ${ }^{32}$.

41 L'agressivité de René Maran ou son cynisme, les paradoxes de l'écrivain comme homme et auteur, ami et amoureux, auteur de la préface de Batouala et agent colonial qui se vante de ramener l'ordre parmi les rebelles, restent à éclairer comme ce flux pulsionnel 
qui échappe au moi conscient. Notre thèse, s'appuyant sur le legs de Jean Bellemin-Noël, est que seule l'étude de l'inconscient, théorisée par la psychanalyse, peut nous faire avancer dans la compréhension de la violence faite à l'Autre. L'œuvre de l'auteur de Batouala l'accepte comme une issue, un soulagement et la décrie en même temps comme un aveuglement. Son écriture de l'intime, à laquelle elle consacre une large place, dans les poèmes, les romans et nouvelles et la correspondance avec Gahisto, montre, et c'est là sans doute que réside son apport inédit, le Moi littéraire comme une instance psychique qui ne peut être que tournée vers le corps.

\section{BIBLIOGRAPHIE}

Les œuvres de René MARAN :

Correspondances avec Manoel Gahisto (références à venir)

Batouala, Paris, Albin Michel, 1921.

Le Livre de la brousse, Paris, Albin Michel, 1934.

Le Cour serré, Paris, Les feuillets bleus, 1935.

Bêtes de la brousse, Paris, Albin Michel, 1941.

Peines de cœur, Paris, Éditions Univers SPLE, 1944.

Un homme pareil aux autres, Paris, Éditions Arc-en-ciel, 1947.

ASSOCIATION LACANIENNE INTERNATIONALE, Une journée avec Édouard Glissant, Paris, Éditions de

l'Association lacanienne internationale, 2009.

BAYARD Pierre, Peut-on appliquer la littérature à la psychanalyse? Paris, Éditions de Minuit, 2004.

BELLEMIN-NOËL Jean, Psychanalyse et Littérature, Paris, PUF, 2012.

BERGSON Henri, Les Données immédiates de la conscience, Paris, Flammarion, 2013.

DE BIASI Pierre-Marc, Génétique des textes, Paris, CNRS éditions, 2011.

DARWIN Charles, L'Origine des espèces, Paris, Garnier Flammarion, 2008.

DUGAS Ludovic, La Mémoire et l'Oubli, Paris, Flammarion, 1917.

FREUD Sigmund, Trois Essais sur la théorie de la sexualité, Paris, 2012, Le Seuil.

- - , L'Inquiétant Familier, Paris, Payot, 2011.

DE GOURMONT Rémy, Physique de l'amour, Paris, Éditions de L'Herne, 2015.

LITTLE Roger, Le Roman d'un nègre à la recherche d'un titre, Paris, Présence Africaine, 2013.

MALINOWSKI B., La Sexualité et sa répression dans les sociétés primitives, Paris, Payot, 1976.

MBEMBE Achille, Politiques de l'inimitié, Paris, La Découverte, 1996.

MELMAN Charles, Lacan aux Antilles, Paris, Éditions Érès, 2014.

RENAULT Matthieu, « Amour de la race ou amour au-delà des races ? F. Fanon, lecteur de René Maran », Paris, Présence africaine, 2013. 
RIFFARD Claire, «Jean-Joseph Rabearivelo et l'intime ", Continents manuscrits, $\mathrm{n}^{\circ} 13$, https://dol.org/ 10.4000/coma.4114.

SANSON Hervé, « Du “tourisme sexuel” à la fusion avec l'autre », Tumultes, n 41, 2013, https:// www.cairn.info/revue-tumultes-2013-2-page-99.htm.

TORT Patrick, L'Effet Darwin, Paris, Le Seuil, 2008.

\section{NOTES}

1. René MARAN, lettre du 10 avril 1914, envoyée de Griko à Manoel Gahisto.

2. Nous nous fondons sur l'édition de L'Herne, datée de 2015.

3. Nous savons que Darwin a lu de Malthus en 1938, Essai sur le principe de population paru en 1798 pour la première fois.

4. René MARAN, lettre envoyée à Manoel Gahisto de Bordeaux, le 11 janvier 1916.

5. Rémy de Gourmont, Physique de l'amour, Éditions de l'Herne, Paris, 2015, p. 6

6. Édouard GLISSANT, voir par exemple Poétique du divers, Paris, Gallimard, 1996.

7. Rémy de GOURMONT, op. cit., p. 10 et 11.

8. Lettre envoyée à Manoel Gahisto le 4 novembre 1915. La ville n'est pas précisée mais il s'agit probablement de Bordeaux. René Maran précise être allée au cimetière sur le caveau familial.

9. Nous savons que René Maran possédait dans sa bibliothèque le livre de Lucien Lévy-Bruhl, La Mentalité primitive (inventaire de la bibliothèque René Maran).

10. Nous devons à Roger LiTTLE la réédition des écrits de René Maran. Nouvelles africaines et françaises, inédites et inconnues, Paris, éditions L'Harmattan, 2018.

11. Nous pouvons remarquer, au passage, l'intérêt de René Maran pour la médecine pratiquée en Oubangui Chari. Il développe ce thème dans Asepsie noire.

12. René MARAN, lettre du 11 octobre 1912, envoyée de Grimari à Manoel Gahisto.

13. Ibid.

14. René MARAN, lettre du 24 mars 1917, envoyée de Fort Sibut à Manoel Gahisto.

15. Id., L'Homme qui attend, op. cit., p. 204.

16. Ibid., p. 205

17. Havelock ELLIS est un psychiatre anglais auteur d'une publication en six tomes: Études de Psychologie sexuelle, Éditions Bibliothèque des introuvables.

18. Patrick tORT, L'Effet Darwin, Paris, Le Seuil, 2008, p. 118.

19. Ibid., p. 120

20. Rémy de Gourmont, Physique de l'amour, p. 7

21. Ibid., p. 16

22. René MARAN, lettre envoyée à Manoel Gahisto de Fort Crampel, le 24 février 1918.

23. Id., lettre envoyée à Manoel Gahisto de Bordeaux, le 11 janvier 1916

24. Id., lettre envoyée à son ami Charles Barrailley, de Bordeaux le 27 octobre 1917.

25. Id., lettre envoyée à Madame Gahisto, de Bordeaux le 9 janvier 1921

26. Frantz FANON, Peau noire, masques blancs. L'homme de couleur et la blanche, Paris, Le Seuil, coll. « essais », 1952. Matthieu RENAUD, spécialiste de Franz Fanon, reprend les termes du débat dans Présence africaine, "Amour de la race ou amour au-delà des races? ", n 187-188, Paris, 2013, p. 231.

27. Matthieu RENAUD, spécialiste de Franz Fanon et professeur à Paris 8 , reprend les termes du débat dans Présence africaine, Amour de la race ou amour au-delà des races ? No 187-188, Paris, 2013, p. 231 
28. Germaine GUEX fait paraître, en 1950, un travail important sur les carences affectives de l'enfant abandonné ou délaissé par ses parents et les conséquences dans la vie de l'adulte d'un tel ressenti. Germaine GUEX, La Névrose d'abandon, Paris, PUF, 1950.

29. René MARAN, Un homme pareil aux autres, op. cit., p. 34

30. Ibid., p. 16.

31. Ibid., p. 39.

32. Achille MBEMBE, Politiques de l'inimitié, Paris, La Découverte, 2016, p. 77.

\section{RÉSUMÉS}

La richesse de l'œuvre de René Maran n'a pas été suffisamment explorée jusqu'à aujourd'hui. La lecture des romans, nouvelles et poésies, mais aussi celle de la correspondance avec Manoel Gahisto nous a amenée à interroger l'influence des théories de Charles Darwin sur la construction des personnages et de l'intrigue de ses romans.

The richness of René Maran's work hasn't been fully discovered so far. The study of the novels, poetry and letters of René Maran to Manoel Gahisto shows the importance of Charles Darwin in René Maran's thought.

\section{INDEX}

Mots-clés : Littérature, instinct, colonialisme, mélancolie, psychanalyse, violence politique, sexualité, Darwin, Rémy de Gourmont.

Keywords : Literature, instinct, colonialism, melancholy, psychoanalysis, political violence, sexuality, Darwin, Rémy de Gourmont.

\section{AUTEUR}

ROSELINE GARCIA BALLESTER

Doctorante, université de Besançon 